\title{
Improved Performance of OFDM by PAPR reduction
}

\author{
Amarish Kumar Soni*1 ${ }^{*}$ Mr. Sachin Kumar ${ }^{2}$ \\ ${ }^{I}$ M.TECH in Electronics \& Communication Enginnering Amity school Of Engineering \& Technology \\ Lucknow , U.P, INDIA \\ ${ }^{2}$ Assistant Proffessor Amity school Of Engineering \& Technology Lucknow, U.P, INDIA
}

\begin{abstract}
OFDM is a special kind of Multi Carrier modulation technique which forms the basis for $4 G$ Wireless system. OFDM is a key broadband technology that can supports data rates in excess of $100 \mathrm{Mbps}$ that is essential for next generation $4 G$ broadband communication.. Peak To Average Power ratio is one of the serious problems in OFDM technology that is problem of extreme excursions of transmitted signal, which reduces the efficiency of transmit high power amplifier. This paper explores the problem of PAPR and focus in reduction of PAPR using $\mu$ law technique. This proposed technique shows the better performance in the presence of non linear high power amplifier by significantly reducing PAPR more than $5.5 \mathrm{db}$, signal to noise ratio is also deceases by $6 \mathrm{db}$ at $B E R=10^{-3}$
\end{abstract}

Keywords: Ofdm ; Papr; BER; Compander; $\mu$ Law

\section{Introduction}

OFDM [1] is frequently referred to as multi-carrier modulation because it transmits signals over multiple subcarriers simultaneously, it can support WLAN data rates up to $54 \mathrm{Mb} / \mathrm{s}$. It uses Fast Fourier transform (FFT) and inverse fast Fourier transform (IFFT) algorithm instead of bank of modulators and demodulators, which allows the multiple subcarriers to overlap yet maintain their integrity. Subcarriers are sent to lower data rates in OFDM ,that's make it to perform robustly in severe multi-path environments. Inter Symbol Interference (ISI) is almost completely eliminated by introducing a guard time between every OFDM symbol, As a result, this modulation approach is being widely adopted in 5-GHz WLAN implementations [2].

For wireless applications, an OFDM based systems are demanding because it provides greater immunity to multipath fading and impulse noise, and the need for equalizers is also eliminated, while using Fast Fourier Transform (FFT) Technique hardware implementation can be realized efficiently.

The basic concept of OFDM is to divide the total bandwidth into sub narrow band by splitting single carrier into multicarrier also called subcarrier (because it occupies smaller band), which are transmitted in parallel. That's why its spectral efficiency is high than conventional single carrier communication system.

The most concerned issue with OFDM which are preventing it for implementing in next generation $4 \mathrm{G}$ wireless communication are its high PEAK TO AVERAGE POWER RATIO. A high peak power in the OFDM signal will occur when most of the sub-carriers align themselves in phase.

The PAPR value is directly proportional to number of subcarrier used in OFDM signal, given by: PAPR $(\mathrm{db})=10 \log (\mathrm{N})$

Where $\mathrm{N}$ denotes the no. of subcarrier

Because of occurrence of high peaks in OFDM signal, power amplifier must be oversized in terms of its average power requirement, traditional OFDM modems are not power efficient, since its DC power consumption is determined by the peak power level, efficiency of the Power Amplifier will suffer dramatically.

To put this in perspective, when PAPR of $10 \mathrm{db}$ occur in OFDM signal, the maximum power efficiency of a Class B power amplifier drops from $78.5 \%$ to $7.85 \%$. Hence there are a loss of DC power consumption of $1.3 \mathrm{~W}$ when $100 \mathrm{~mW}$ power level is desirable. This very high DC power consumption is one of the main obstacles for the practical implementation of OFDM modulation for portable applications, including 5GHz WLAN enabled devices such as smart phones, palmtop laptops and personal digital assistants. [3]

The OFDM modulation scheme also requires highly linear up converters due to its high peak-toaverage power ratio. And due to high PAPR, up converter must have a high-level compression point, which results in high DC power consumption.

Finally, To sustain high power consumption, and thereby increasing the component cost , semiconductor manufacturers will have to use more expensive packaging design to compensate high PAPR problem.

Therefore to meet consumer expectations for user autonomy (long battery life in portable applications), there are need to work on designing low power consumption that's why manufacturers are specifying low power consumption as part of their system requirements. But, merely specifying a change 
in power level mean does not solve the problem. So this is a challenging task for the OFDM modem designers to achieve acceptable levels of power consumption in their designs. Until then, the penetration of WLANs and next generation communication system like 4G using OFDM will likely be limited to high-end, low-volume products. [3]

In this paper, we suggest very effective technique for reducing the PAPR of OFDM signals. That are based on the properties of the $\mu$-law to reduce dynamics range of the signal .

The paper is organized as follows: In Section II, the PAPR in OFDM is introduced. Section III, The technique will be explained. Section IV, simulation results will be made. Section V, conclusions will be done.

\section{Papr In Ofdm Systems}

If we consider $\mathrm{N}$ modulated data symbols from a particular signaling constellation, $\mathrm{X}_{\mathrm{K}}=(\mathrm{X} 0, \mathrm{X} 1, \ldots$, $\mathrm{X}_{\mathrm{n}-1}$ ), over a time interval $[0, \mathrm{~T}]$, the OFDM symbol can be written as

$$
\begin{aligned}
& X(t)=\sum_{k=0}^{n=-1} x_{k} e^{j 2 \pi k f_{0} t} \\
& \text { Where } f_{0}=\frac{1}{T}
\end{aligned}
$$

The discreet time version is obtained by replacing $\mathrm{t}=\mathrm{n} . \mathrm{T}_{\mathrm{b}}$ and $\mathrm{T}_{\mathrm{b}}=\mathrm{T} / \mathrm{N}$

$$
X_{n}=\sum_{k=0}^{N=-1} x_{k} e^{j 2 \pi k n / N}
$$

The peak to average power ratio is then given by:

$$
\text { papr }=\max _{\mathrm{o} \leq \mathrm{t} \leq \mathrm{T}} \frac{[\mathrm{x}(\mathrm{t})]^{2}}{\mathrm{E}\left[[\mathrm{x}(\mathrm{t})]^{2}\right]}
$$

Which is ratio of peak instantaneous power to average power where $\mathrm{E}$ [.] is the expectation ratio.

In practice, during signal transmission the occurrence of these large fluctuations in OFDM signal causes the large PAPR requires very high linear dynamic range and inefficient hardware design and also affects BER during OFDM signal transmission.. The PAPR ratio measures the dynamic range in OFDM signals. So high PAPR induces a high dynamic range which shows high variability in the signal range. The large PAPR levels increases the implementation complexity like the number of quantization bits of the Analog to Digital and the Digital to Analog converters such that large peaks of composite signal can be represented with good precision. To avoid any information loss, these large dynamic ranges must be compensated by the hardware design which is practically very tough task and seems to impossible. Because high PAPR signals generally exhibit high energy concentrations over small portions of the signal, the designing of hardware to compensate for only a small fraction of the signal leads to increase complexity, inefficiency in design and implementation costs.

Non linear distortions is another major problem which is produced by OFDM signals because of its high PAPR. High power amplifiers at the transmitters side will show effective performance only when OFDM signals are within the dynamic range which are passed through the amplifier. When amplifiers limit the transfer of large induced peak powers in signals, amplifiers are then forced to operate in the non-linear regions creating non-linear distortions which causes out of band radiation which influences BER performance. The ideal condition is that the power amplifier is intuitively recoverable to their original form. if distortions are nonlinear, the changing in signals is no longer predictable or recoverable. [4]

In addition, when PAPR problem occurs the choices of power amplifier and up-converters will also be crucial. As a result, power efficiency is decreased significantly due to PAPR problem. For example, the maximum power efficiency of a Class B power amplifier drops from $78.5 \%$ to $4.6 \%$, when the PAPR increases $0 \mathrm{~dB}$ to $17 \mathrm{~dB}$, as stated in the IEEE 802.11 a standard. Power efficiency is pivotal in mobile communications such as laptop and PCs.

\section{Description Of The Technology Used To Reduce Papr}

There are at least two ways to reduce the PAPR as defined in equation (3). Firstly the numerator can be made variable. The objective is to reduce the peak power of the transmitted signal as much as possible to achieve the overall reduction of PAPR. Some techniques that are based on this motivation deal with signal clipping and filtering to reduce the peak powers of the transmitted signal. On the other hand, the PAPR can be reduced by increasing the denominator. To do that new technique will be suggested. This technique depends on a simple logarithmic computation that can only handle a positive signal. The logarithm computation is known as a compressor. 
The logarithm computation is known as a compressor. Computation of a compressor is called an expander. The combination of a compressor and expander is called a compander (compress and expand) [5].

The two most popular compressing functions used worldwide are $\mu$-law and A-law. In this paper we will use these two companders to reduce PAPR in OFDM which are described below:

\section{A. $\quad \mu$-law Compander}

The compression characterstics for $\mu$-law is :

$$
\mathrm{g}(\mathrm{x})=\frac{\log (1+\mu|\mathrm{x}|)}{\log (1+\mu)} \operatorname{sign}(\mathrm{x})
$$

where $\mathrm{x}$ : input signal .

$\mu$ : parameter controls the amount of compression . Title

\section{B. A-law Compander}

The characterstic of this compander is given by :

$$
g(x)=\left\{\begin{array}{ll}
\frac{1+\ln A|x|}{1+\ln A} \operatorname{sgn}(x) & \frac{1}{A} \leq|x| \leq 1 \\
\frac{A|x|}{1+\ln A} \operatorname{sgn}(x) & 0 \leq|x| \leq \frac{1}{A}
\end{array}\right\}
$$

A : parameter controls the amount of compression .

In this paper, we use the compression after the IFFT process at the transmit end and expansion prior to FFT process at the receiver end. The block diagram of digitally companded OFDM system shows in Fig. 1.

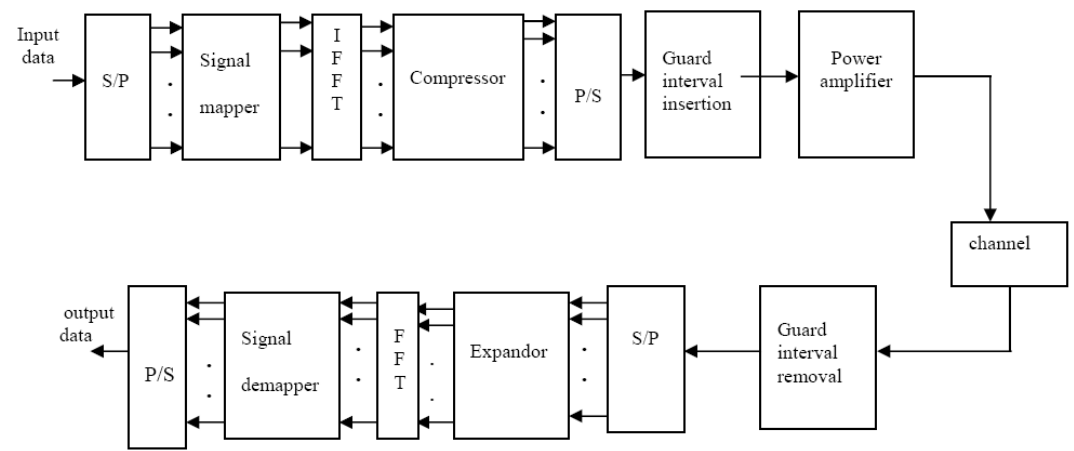

Figure 1: OFDM system with companding technique for PAPR reduction [6]

A $\mu$-law companding scheme to reduce the PAPR of OFDM signal was first proposed by Wang et al, In [7]. According to this scheme, to produce a companded OFDM signal $x_{\mu c}$, the time domain OFDM signal (x[n]) is applied to a $\mu$-law compressor given by

$$
\mathrm{x}_{\mu \mathrm{c}}=\frac{\operatorname{Asgn}(\mathrm{x}|\mathrm{n}|) \ln \left[1+\left|\frac{\mathrm{x}[\mathrm{n}]}{\mathrm{A}}\right|\right]}{\ln (1+\mu)}
$$

where, $\mu$ is the parameter controlling nonlinearity of the companding function and $\mathrm{A}$ is the peak value of the OFDM signal before companding. $\ln ($.$) and \operatorname{Sgn}($.$) are the standard natural and signum logarithmic function$ respectively. The discrete time domain companded OFDM signal is first converted to analog signal using D/A converter and then amplified using High Power Amplifier (HPA) to achieve the desired power level. The amplified signal is then transmitted over the communication channel. At the receiving end, to recover the transmitted time domain signal, the received signal $(\mathrm{r}[\mathrm{n}])$ is applied to the expander. The time domain OFDM signal at expander output is given by

$$
\mathrm{X}_{\mu \mathrm{e}}=\frac{\mathrm{A} \exp \left\{\frac{\mathrm{r}[\mathrm{n}]}{\mathrm{Asgn}(\mathrm{r}[\mathrm{n}])} \ln (1+\mu)\right\}}{\mu \operatorname{sgn}(\mathrm{r}[\mathrm{n}])}
$$

Here, the received signal $(r[n])$ is nothing but the companded OFDM signal $\left(\mathrm{x}_{\mu \mathrm{c}}[\mathrm{n}]\right)$ corrupted by quantization and channel noise. Before companding, the real and the imaginary parts of the complex time domain 
OFDM signal have Gaussian distribution. But, after applying the $\mu$-law companding scheme, the PDF of real and imaginary parts no longer remains Gaussian distributed. Fig. 3 shows the PDF of real part before and after companding of OFDM signal.

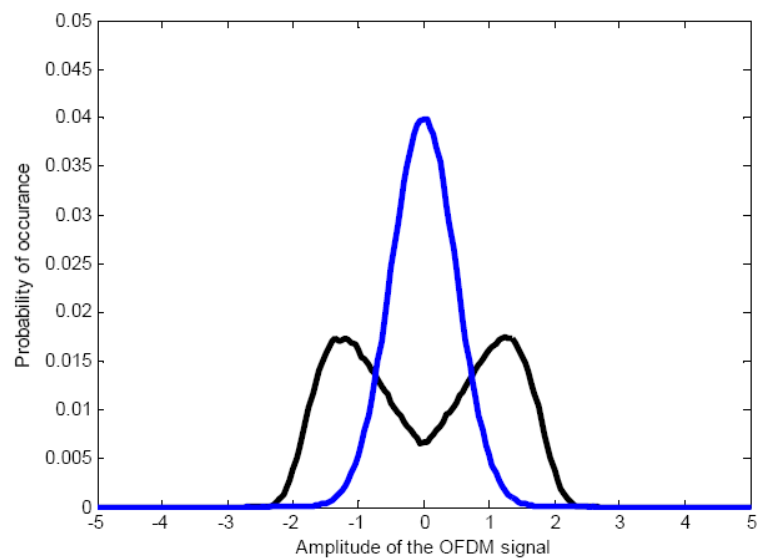

Figure 2: PDF of real part of original and $\mu$-law companded OFDM signal amplitude

\section{Simulation Results}

Simulations are used to clarify the PAPR reduction capability and BER performance with the companding technique. Here we used $\mathrm{N}=256$, 512 , and 1024 sub carriers using QPSK ,16 QAM, and 64 QAM respectively. Rapp's solid state power amplifier model (SSPA) [8],[9] for the high power amplifier (HPA) are used with the characteristic given by:

$$
\mathrm{v}_{\text {out }}=\frac{\mathrm{v}_{\text {in }}}{\left\{1+\left(\frac{\left|\mathrm{v}_{\text {in }}\right|}{v_{\text {sat }}}\right)^{2 p}\right\}^{1 / 2 p}}
$$

Where, vin $=$ the complex input, vout $=$ complex output signals . vsat $=$ output saturation level .

The parameter p, also called "knee factor," controls the smoothness of the characteristic. We also define the input back-off (IBO) w.r.t the saturation values:

$$
\text { IBO }=10 \log _{10}\left\{\frac{\mathrm{v}^{2} \text { sat }}{\mathrm{E}\left|\mathrm{v}_{\mathrm{in}}\right|^{2}}\right\}
$$

In this paper, we considered a rapp model HPA with knee factor $\mathrm{p}=2, \mathrm{IBO}=3.5 \mathrm{~dB}$ and used Additive White Gaussian Noise (AWGN) channels to evaluated the BER performance.. CCDF and BER performances will be investigated In the following :

\section{A. CCDF Performance:}

A CCDF curve shows the time spent by a signal at or above a given power level (expressed in $\mathrm{dB}$ ) relative to the average power. A CCDF curve plots relative power levels against probability,[5,6] and can be explained with a set of data having the probability density function (PDF). To obtain the CCDF first we compute Cumulative Distribution Function (CDF) by taking integral of the PDF, then inverting the CDF results . It means that $\mathrm{CCDF}=1-\mathrm{CDF}$.

PAPR statistics are given in terms of the CCDF. The CCDF shows the probability of an OFDM frame exceeding a given PAPR ,

$\operatorname{CCDF}(\operatorname{PAPR}(\mathrm{x}))=\operatorname{Pr}(\operatorname{PAPR}(\mathrm{x})>\operatorname{PAPR} 0)$.

Figs. 3 shows the performance improvement of the new scheme over a original OFDM (without peak reduction) system for the $\mu$ - law. PAPR is reduced by almost $5.8 \mathrm{~dB}$ smaller than without PAPR reduction when the probability goes to $10^{-3}$ in this scheme the same number of frame will be sent for each modulation. However, The same improvements can be obtain by using A-law compander.

\section{B. BER Performance:}

The BER performance of OFDM is evaluated using computer simulation. In this study, the channel is assumed to be known perfectly at the receiver. The modulated signal is affected by SUI multipath channels [10] and AWGN.

The BER performances of OFDM systems over the AWGN channel are given by the BER curves in Fig We see that $\mathrm{Eb} / \mathrm{N} 0$ required for $\mathrm{BER}=10^{-4}$ is improved by $6 \mathrm{~dB}$. The BER performances of OFDM systems over the AWGN channel shown in Fig 4. The improvements that are seen in BER in proposed system which includes 
the amplifier is just another way of visualizing the PAPR reduction. The achievement of PAPR reduction that was our goal, means the amplifier is operating more in its linear region, which means we don't see as many errors related to clipping from saturation region of power amplifier. In other words, the dominant effect that is causing the errors is the amplifier and not channel. The proposed technique reducing that and thus it makes sense that we are seeing a lower BER.

\section{Conclusions}

This paper, presents a better technique for PAPR reduction of OFDM signals. This technique uses the $\mu$ law or A-law compression with optimum values of $\mu$ and A here 255 for $\mu$, which gives better performance , where the $\mu$-law and A-law reduces dynamics range of the signal. Increasing the compression (via the companding parameter, $\mathrm{u}$ ) reduces the

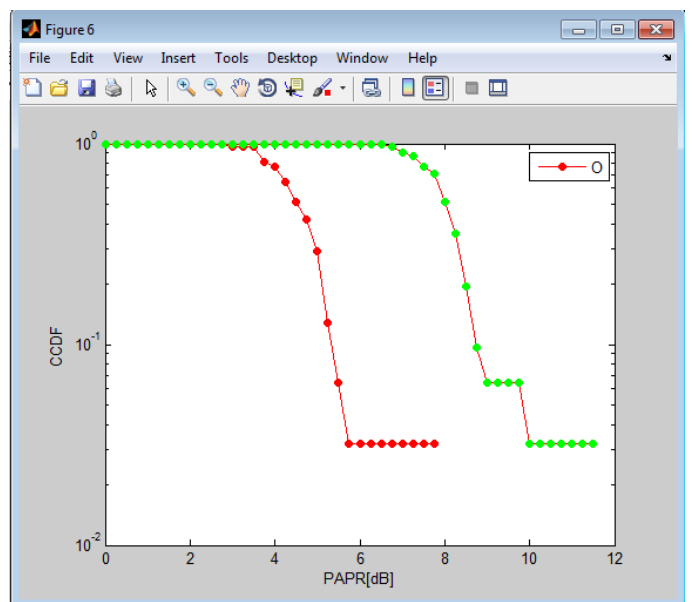

Figure 3: comparison of CCDF of PAPR with the proposed technique to the without companded OFDM signal.

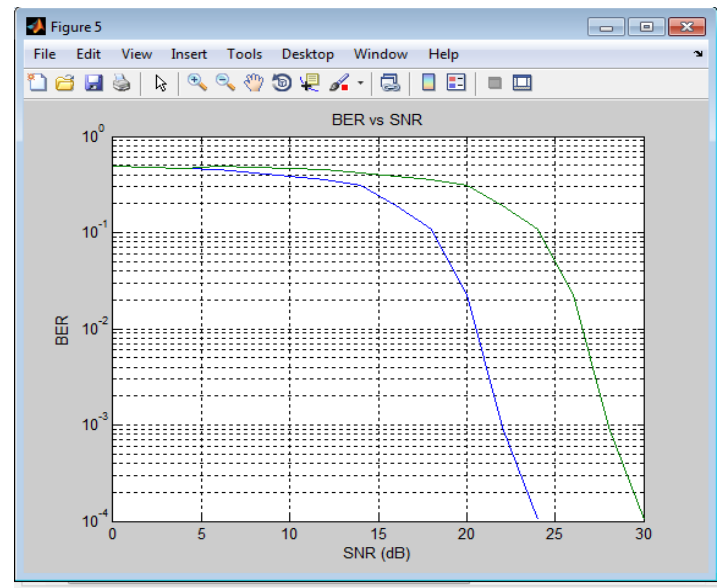

Fig. 4. BER performance with the proposed technique over AWGN beneficial

Non-linear distortion and suggests that moving the operating point towards saturation might be

The main advantage of this technique the reduction of PAPR more than $5.8 \mathrm{~dB}$ and decreasing the BER over original system where the new technique is evaluated in presence of nonlinear power amplifier

\section{References}

[1] R. V. Nee and R. Prasad,_ OFDM for wireless multimedia communications, Norwood, MA: Artech House Publishers, January 2000.

[2] Peng Liu "A New Peak to Average Power Ratio Reduction Schemes for OFDM System "M. Eng. thesis, Concordia university, Canada , Jan. 2004.

[3] OFDM challenge,EE TIMES Jim Wight 10/23/2001 03:05 PM EDT

[4] J. A. Davis and J. Jedwab, "Peak-to-mean power control in OFDM, Golay complementary sequences, and Reed-Muller codes," IEEE Trans. Inform. Theory, vol. 45, pp. 2397-2417, Nov. 1999.

[5] J . G . Proakis and M . Salehi, Communication systems engineering, 1994 by prentice-Hall ,Inc.

[6] H. Sakran\#1, M. Shokair \#2, A. Abou Elazm \#3 "A New Peak-to-Average Power Reduction Technique in the OFDM System Using $\mu$-Law Compander', IEEE ISWCS 2008. 
[7] Wang X. B., Tjhung T. T., Ng C. S., "Reduction of peak-to-average power ratio of OFDM system using a companding technique", IEEE Transactions on Broadcasting, vol.45, no. 3, pp. 303-307, Sept. 1999..

[8] C. Rapp, "Effects of the HPA-nonlinearity on a 4-DPSK/OFDM signal for a digital sound broadcasting system," Tech. Conf. ECSC'91, Luettich, October 1991.

[9] F. H. Gregorio "Analysis and Compensation of Nonlinear Power Amplifier Effects in Multi Antenna OFDM Systems “, Dr. Eng. thesis,_Helsinki University of Technology,_Finland, Nov. 2007.

[10] V. Erceg, K.V.S. Hari, M.S. Smith, D.S. Baum et al, "Channel Models for Fixed Wireless Applications ", IEEE 802.16.3 Task Group Contributions, Feb2003.

[11] W. Jeon, K. Chang,and Y. Cho," An Equalization method for Orthogonal frequency division multiplexing system in Time-Variant Multipath Channels "IEEE transaction on communication, VOL. 47 NO. 1, JANUARY 1999.

[12] J. Rinne and M.Renfors," An Equalization method for Orthogonal frequency division multiplexing system in channels with multipath propagation frequency offset and phase noise" IEEE,1996.

[13] H. Sari, G. Karam, and I. Jeanclaudle " Frequency-domain equalization of mobile radio and terrestrial broadcast channels " IEEE conference, 1994 . 